\title{
Vascular aging and subclinical atherosclerosis: why such a "never ending" and challenging story in cardiology?
}

This article was published in the following Dove Press journal:

Clinical Interventions in Aging

23 August 2017

Number of times this article has been viewed

\author{
Stela lurciuc ${ }^{1,2}$ \\ Anca Maria Cimpean ${ }^{3}$ \\ Florin $\mathrm{Mitu}^{4}$ \\ Rodica Heredea ${ }^{5}$ \\ Mircea lurciuc ${ }^{1,2}$ \\ 'Department of Preventive Medicine, \\ Angiogenesis Research Center, "Victor \\ Babes" University of Medicine and \\ Pharmacy, ${ }^{2}$ Clinical Emergency County \\ Hospital "Pius Brînzeu," 'Department \\ of Microscopic Morphology/Histology, \\ Angiogenesis Research Center, \\ "Victor Babes" University of Medicine \\ and Pharmacy, Timisoara, ${ }^{4}$ Department \\ of Cardiology, "Grigore T Popa" \\ University of Medicine and Pharmacy, \\ lasi, ${ }^{5}$ Department of Pathology, \\ Louis Turcanu Children Hospital, \\ Timisoara, Romania
}

Correspondence: Anca Maria Cimpean Department of Microscopic Morphology/ Histology, Angiogenesis Research Center, "Victor Babes" University of Medicine and Pharmacy, Piata Eftimie Murgu 2, 30004I Timisoara, Romania

Tel +40 720060955

Email ancacimpean1972@yahoo.com
Abstract: The true onset of atherosclerosis remains one of the biggest challenges for cardiologists. Is atheroma plaque development considered the earliest step of vascular aging? If so, when it starts? Before or after birth? If it starts before birth or early during childhood, it seems that Thomas Sydenham was right: "A man is as old as his arteries." Except disorganization of elastic fibers, less is known about the morphology of vascular aging and also about the molecular events influencing the age of arteries, arterial stiffness, and their role in the appearance of future complications. Cellular and molecular events responsible for the switch from physiologic to pathologic aging of human arteries are less known. Epigenetic, genetic, and environmental influences at the onset of early vascular aging (EVA) should specifically influence the process. This paper briefly reviews the controversial data regarding vascular aging with an emphasis on the less known facts about the morphology of EVA.

Keywords: arterial stiffness, blood pressure variability, arterial aging, telomeres

\section{Age and pulse: “oldies" but still controversial "goldies"}

Pulse wave velocity is not a new topic in the field of cardiovascular pathology. This issue was mentioned early in the literature, in ancient papers such as the Huangdi Neijing from the Han Dynasty period. Wang Shuhe (born in $180 \mathrm{CE}$ ) was a Chinese physician who wrote the Maijing (The Pulse Classics), an influential work describing the pulse and its importance in the diagnosis of diseases. The physician had three specific spots at the wrist used to assess the quality and quantity of the pulse. "Recently" (if we could say so), in 1854, German physician Karl von Vierordt created a device called a sphygmograph, ${ }^{1}$ but it is well known that a British physician, Frederick Henry Horatio Akbar Mahomed (1849-1884) had previously described pressure pulse features in patients with high blood pressure and arteriosclerosis consequent to aging. ${ }^{2}$ Nowadays, many devices are able to evaluate pulse pressure and wave.

European Society of Cardiology Guidelines highlighted the importance of the pulse wave and the reflected wave measurement in order to assess the vascular age. Ductus arteriosus involves a series of subclinical (endothelial dysfunction) to clinical level (atherosclerotic plaque and target organ damage) alterations including changes in large arteries, elastic type, like aorta, and also in small muscular arteries like peripheral arteries type. Both microvascular and macrovascular beds are affected, promoting hypertension and arterial stiffness development. ${ }^{3}$ There are still different opinions regarding the timing of onset of arterial stiffness, and questionable issues such as 
whether this happens before or after the onset of hypertension still persist. Large artery stiffening (aortic, mainly) and wave reflection have been identified as being an exceedingly important pathophysiological determinant of isolated systolic hypertension and of pulse pressure increasing with age.

\section{Early vascular aging (EVA): how "early" we define it and what criteria we use?}

Vascular aging is described as a gradual process involving biochemical, enzymatic, and cellular events in vascular area combined with epigenetic and molecular alterations, and it is considered that arterial aging is a fundamental reflection of biological aging in general and a determinant of organ function. In the arterial wall, this is characterized by a decrease of elastin content, as well as by the production and accumulation of "bad" collagen and its cross-linkages, leading to increased arterial stiffness and elevated central blood pressure as well as brachial blood pressure, accompanied by increased variability in systolic blood pressure (SBP). A better understanding of these processes has led to the proposal of a condition named EVA in patients with increased arterial stiffness for their age and sex. This is a condition that could increase cardiovascular risk, and it is associated with various degrees of cognitive dysfunction, as well as other features of biological aging. ${ }^{4}$

EVA seems to be useful for the clinical practice management regarding individuals with cardiovascular risk or strong genetic inheritance of premature cardiovascular disease. Increased arterial stiffness, dilated elastic arteries of central type, impaired endothelial function, and vasodilation are main aspects of this process. Countering this phenomenon can be made with the help of aggressive decrease of atherosclerosis modifiers, but there is no strong evidence to that effect. ${ }^{5,6}$

\section{Determinants of vascular aging: from morphology to molecular phenotype and beyond!}

It is considered that vascular aging is found from several and sequential alterations that lead to the replacement of elastin fibers with collagen fibers in the vessel wall, which forms a less elastic structure due to the collagen bridges that prevent their elongation. This is the so-called physiological arterial stiffness. In time, there may be a pathological aging, consisting of various types of plaque deposition. ${ }^{7}$ There is no well-defined criteria that characterize EVA in this moment. What seems to be "early" for clinicians may be "too late" stages of vascular aging for vessel wall and also for the patient. "Early" microscopic changes in the structure of the arterial wall do not overlap with "early" vascular aging definition and assessment at all! Previously reported data of Napoli et al about the microscopic changes in the aorta wall in human fetuses seem to be neglected while defining EVA not because it gave less importance to this issue but because of the lack of methods assessing the early steps of vascular aging in prenatal life. ${ }^{8}$ Data regarding the assessment of early steps of aortic wall changes and atheroma plaque formation are scattered for prenatal period, childhood, and teenage. It is suggested that EVA starts in fetal life as stated by few papers reporting perinatal atherosclerotic lesions in the fetal coronary arteries of babies of mothers who are smokers ${ }^{9}$ or by data suggesting the involvement of telomere length influencing arterial function and aging properties which could be programmed during fetal life or influenced by adverse growth patterns in early postnatal life. ${ }^{4}$ An accurate correlation between microscopic and clinical criteria should be mandatory for a better assessment of subclinical atherosclerosis and EVA. The guideline including maternal and fetal risk criteria should be implemented as early as possible for a better evaluation of vascular aging. Recently launched first precision diagnostic biomarker, "knowPLAQUE"T," for the detection of atherosclerotic coronary artery disease by using blood sample, seems to be a valuable tool for the early assessment of vascular aging and subclinical atherosclerosis risks (http://www.prnewswire.com/news-releases/globalgenomics-group-announces-breakthrough-diagnostic-bloodtest-for-plaque-detection-in-the-heart-300425836.html).

The mechanism of vascular aging is associated with changes in the mechanical and structural properties of the arterial wall. These changes lead to the loss of arterial elasticity and reduced compliance because of the changes in the ratio between elastin and collagen fibers. Over time, destruction of elastin fibers with linear parallel structure takes place, due to the so-called fatigue phenomenon of the elastin. This leads to the breaking and fragmentation of elastin fibers which are replaced in a higher ratio with collagen fibers, resulting in a structure with increased rigidity. The "fatigue of the elastin" phenomenon is more significant as the frequency and amplitude of demands are higher, that is, pulse wave frequency and pulse wave amplitude. ${ }^{10,11}$

The beginning of elastic fiber loss or damage remains a questionable issue for cardiologists. Our preliminary data showed that disorganization of elastic fibers appears early in human fetal aorta and continues during postnatal life, being extended immediately after birth (Figure 1A and B). 

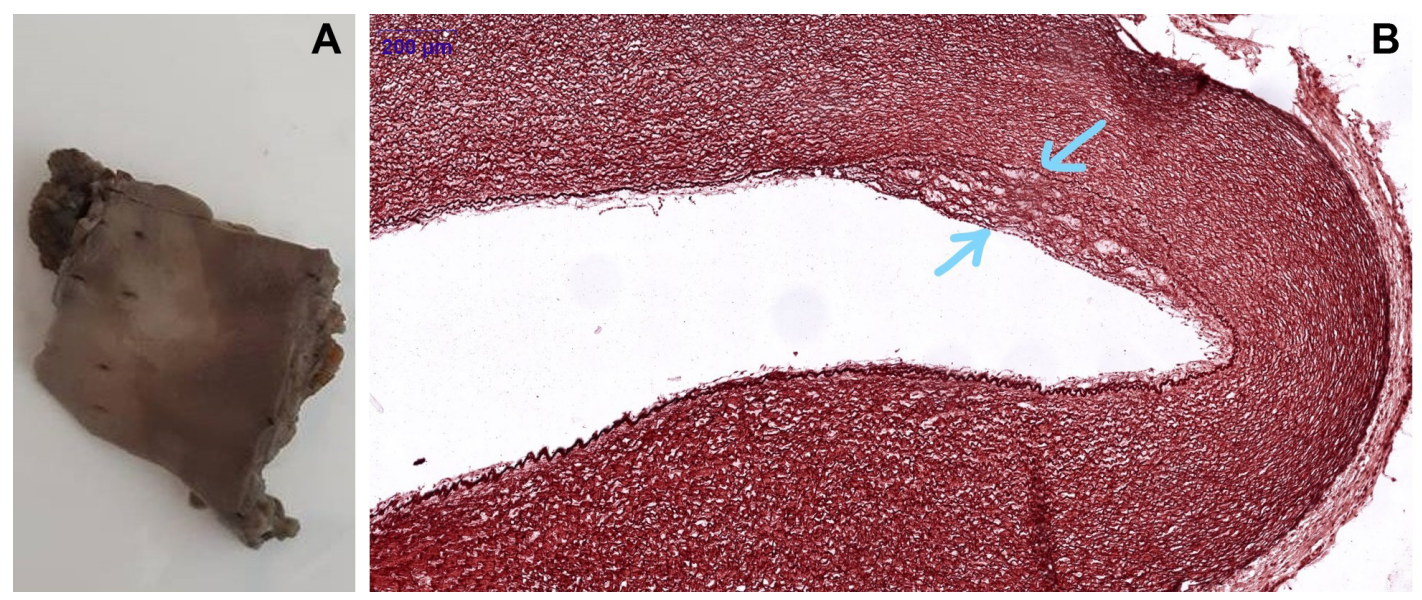

Figure I Aorta of I-day-old human neonate showing macroscopically detected fatty streaks (A) certified as having the disorganization of elastic fibers (B, inset, orcein stain, $\times 400)$ and lipid accumulation.

The process is not homogeneous along all length of the fetal or neonatal aorta, being mostly and easily detected on aortic arch level in prenatal life and immediately after birth. In this context, a big challenge is launched: how and when to define EVA related to detected microscopic changes which, for sure, anticipate the beginning of clinical vascular aging. Due to these facts, arterial stiffness may be seen as a complication of true, microscopic EVA. Molecular and genetic assessment should be mandatorily included in the criteria of vascular aging definition.

Determinants of EVA can be classified as prenatal and postnatal factors. Prenatal factors are related to mothers' behavior regarding food, smoking, or alcohol consumption. Some of the genetic mutations from prenatal life seem to influence the development of EVA in children, by starting even from intrauterine life without having clinical or subclinical manifestations in childhood.

Now, we do not have well-established criteria to characterize an EVA in newborn and infants. Also, the follow-up of children for such data may be strange for their parents who do not really understand the importance of such procedures for an apparent healthy child. For example, literature reported that psycho-social factors related to EVA are scattered. ${ }^{12}$ In a recent experimental study performed by Rana et al, ${ }^{12}$ it was discussed that trait aggression was followed by increased wall-to-lumen ratio in the thoracic aorta, increased sensitivity to phenylephrine-induced vascular contractility, and increased norepinephrine content in the heart. Maternal hypoxia favors the appearance of a rapid weight gain during childhood and may induce early changes inside the vascular walls. In a comparative study performed by Wang et al, ${ }^{13}$ four main criteria such as maternal hypoxia, sex, hyperlipidemia, and postnatal hypoxemia were used to assess their impact on morphometric parameters of the thoracic aortas in the offspring of Sprague-Dawley rats. Aortas from rat babies delivered from mothers with hypoxia exhibited lesions similar to early events in atherosclerosis related to endothelial cell damage, thickening and collagen deposition inside intima, inflammatory cell infiltration of sub-endothelial space, and migration and proliferation of vascular smooth muscle cells to the intima. Thus, the research group concluded that maternal prenatal hypoxia induced early vascular changes inside aorta and increased the risk of hyperlipidemia appearance during postnatal life.

With age, due to the occurrence of some triggers, an increase in the migration of vascular smooth muscle cells from tunica media to the tunica intima takes place and also their proliferation is enhanced. Over time, there appears an augmentation of the homocysteine levels and an activation of genes that induce metalloproteinase imbalance. Also, a growth in wall shear and oxidative stress, active and sustained inflammation, reduced the levels of nitric oxide in the endothelium; they all lead to the development of endothelial dysfunction. ${ }^{14,15}$

It seems that there is a genetic programing for vascular damage that promote arterial stiffening over time. Some authors believed that telomeres have a particular role in vascular aging steps. Telomeres are repetitive TTAGGG hexanucleotide monomers with size ranging from 12 to $7 \mathrm{kbp}$ (kilo base pairs) which deteriorate (become shorter) to the limit of replicative senescence called Hyflick limit. Shortening over this limit involves the degradation of genetic information and the inability of cell division. They shorten with age in each individual. Young people have telomerase, 
which protects telomeres from shortening. Many researchers considered progressive telomere shortening as a marker of an individual's aging in general and especially a marker of arterial aging.

DNA damage is the main determinant of vascular aging. Accumulation of damaged DNA is associated with a rapid functional decline of the vasculature. ${ }^{16}$ Shortening of telomere length is certified to be associated with EVA. Telomere length may predict the earlier stages of endothelial cell senescence and initiation of the atherosclerotic process. ${ }^{17}$ Coimbra et al suggested that childhood adversity (violence, low socioeconomic status, maternal depression, family disruption, and institutionalization) accelerates shortening of telomere length and induces an accelerated EVA followed by increased prevalence of hypertension during adult life. ${ }^{18}$

Latest studies showed that there is an inverse correlation between telomere length and arterial stiffness measured by pulse wave velocity (PWV) and arterial age, respectively. ${ }^{19-21}$ The mechanisms involved in vascular aging lead to changes such as lumen dilation, intima-media thickness ratio changes, endothelial dysfunction, calcifications, reduced arterial compliance in small artery and then large artery, impaired angiogenesis, and development of collateral vessels after arterial obstruction. ${ }^{20}$ Vascular age is advanced in obese children with cardiovascular risk factors and in patients with familial dyslipidemia. Vascular age is far gone in obese children with high levels of fasting triglycerides. ${ }^{21}$

According to some authors, arterial stiffness is one of the most important changes in arterial aging process. Carotidfemoral PWV (cfPWV) is considered the "gold-standard" measurement of arterial stiffness. PWV can be determined at the following levels: large arteries, central, elastic type, represented by aortic cfPWV; peripheral arteries of muscular type, like the limb arteries, carotid-radial PWV, or femoralposterior tibial PWV. Carotid-posterior tibial PWV is a factor of combined stiffness of large vessels (elastic type) with peripheral vessels (muscular type). In most studies, stiffness is assessed by cfPWV because it is best correlated with overall mortality and cardiovascular mortality. ${ }^{22}$ In recent years, several studies address cfPWV parameter. It is important to evaluate arterial stiffness on target levels of blood pressure recommended by European Society of Cardiology (ESC) because it is known that any increase in blood pressure induces the enhancement of arterial stiffness, but this is not a linear growth. Any drug that reduces blood pressure reduces arterial stiffness also. For this reason, it is useful to assess arterial stiffness corresponding to target levels recommended by the ESC. Latest publications (consensus in arterial stiffness) define reference values for arterial stiffness and cfPWV for different age groups and for different degrees of hypertension. By the assessment of arterial stiffness in healthy people and people with cardiovascular risk, it has been shown that cfPWV increases with age: from $5.5 \mathrm{~m} / \mathrm{s}$ at 10 years to $11 \mathrm{~m} / \mathrm{s}$ at 80 years, with a rapid increase around 55 years. ${ }^{23}$

cfPWV measurement is a simple, robust method and easy to implement in clinical practice. cfPWV has demonstrated an added predictive value for cardiovascular events in various populations, beyond ESC-SCORE, ESH-ESC Risk Chart, and Framingham risk score. cfPWV is listed among subclinical organ damage by the ESH-ESC Guidelines for the management of hypertension. Its value as a surrogate endpoint remains to be demonstrated by therapeutic intervention trials. It is important to use this type of surrogate endpoints in future clinical trials for the evaluation of various pharmaceutical products. They should be useful not to exclusively decrease blood pressure but also to be acting on arterial stiffness, vascular remodeling, and upon other determinants of subclinical atherosclerosis.

Our experience in this field shows delayed arterial stiffening using sustained physical training. ${ }^{24}$ These effects are maintained as long as a high level of exercise and physical training are continued and are cancelled once the training is stopped, leading to further stiffening. In cardiac rehabilitation program, every effort is important, even one with shorter duration; it has a cumulative effect on hemodynamic parameters and arterial stiffness. ${ }^{25,26}$ There are some conflicting studies regarding stiffness in hypertension; some authors consider that initially increased stiffness led to high blood pressure, and others believe conversely; however, one thing is clear, that is, both the mechanisms are self-sustaining in a subject with uncontrolled blood pressure.

Pulsating wave is generated at the root of the aorta, runs with high speed in a stiff vessel, reflects in the periphery, and enhances central systolic pressure. This mechanism is the main topic of several studies showing a correlation between arterial stiffness and augmentation index, augmentation pressure, central arterial pressure, intima-media thickness, and volume of the plaque. ${ }^{27}$

Increased arterial stiffness leads to elevated central blood pressure as well as brachial blood pressure, accompanied by increased SBP variability. ${ }^{4}$ In the assessment of hypertensive patients, it is essential to establish SBP variability. Various additional data may be derived from ambulatory blood pressure monitoring recordings. They include blood 
pressure variability, morning blood pressure surge (MBPS), blood pressure load, and the ambulatory arterial stiffness index (AASI). However, their additional predictive value is not clear yet. Lately, the research is focused on the assessment of nocturnal blood pressure and the treatment of hypertensive patients with subclinical atherosclerosis. This parameter is useful in evaluating blood pressure parameters as in diurnal/nocturnal type: Dipper/Non-D/Extreme-D/ Reversed-D and also determining whether it is sustained/ isolated nocturnal/isolated diurnal hypertension. We can agree that the MBPS should be a therapeutic target for preventing cardiovascular events in hypertensive patients, and the drugs should provide 24-hour efficacy. However, because an MBPS may also be harmful for normotensive subjects, evaluating the range of early morning blood pressure might be useful for normotensives as well. ${ }^{28}$

Another parameter derived from ambulatory blood pressure monitoring on $24 / 48$ hours is the AASI $=1-\alpha$, where $\alpha$ is the statistical regression slope between diastolic blood pressure and SBP. There are still controversial issues regarding the clinical utility of this criterion representing indirect arterial stiffness. ${ }^{29}$

It is useful to assess the blood pressure variability even for persons with normal blood pressure. There are many factors involved in the variability of blood pressure that are triggers of subclinical atherosclerosis. On short term, this can be calculated by automatic monitoring on 24/48 hours for establishing a blood pressure profile or, on a long term, at home through self-monitoring. Giuseppe Mancia pointed out some of the factors involved in the variability of blood pressure: humoral, endothelial, behavioral, rhythmic influences (probably largely central), vasomotor status, external stimuli, mechanical factors, ventilation; arterial stiffness, genetic factors, baroreflexes. ${ }^{30,31}$ There are a short-term variability (measured by ambulatory blood pressure monitoring within the 24/48 hours) and a long-term blood pressure variability.

There is an increased interest in discussing more about a parameter that would correlate with subclinical atherosclerosis and vascular age. There are some studies about blood pressure dispersion, measured twice daily in those enrolled in the study who underwent the gradual taper of antihypertensive medications. ${ }^{31}$ The elderly have a relatively high variability of the blood pressure due to their high arterial stiffness. We can speculate to determine the dispersion of the blood pressure and heart rate or we can analyze the multiscale entropy of the blood pressure values. ${ }^{32}$

\section{Biomarkers of EVA in clinical practice: where we are and what should be done to implement them in routine clinical practice?}

Except precision diagnostic biomarker "knowPLAQUETM" recently launched, no other single biomarker or combination of biomarkers are applied in clinical practice. The "knowPLAQUE" is the first diagnostic blood test able to detect coronary artery plaque in any stage of its development, and thus, it may be used as a biomarker tool at any age. This mass-spectrometry-based metabolomics diagnostic biomarker is able to detect the signature of atherosclerotic plaque components by a simple mass spectrometry measurement from blood sample.

Anti-aging proteins with direct impact on cardiovascular system, detectable by its soluble form in serum from patients with high risk for EVA, may represent a proper alternative. Deficiency of anti-aging "hormone" Klotho induces medial calcification, intima hyperplasia, endothelial dysfunction, arterial stiffening, hypertension, and impaired angiogenesis and vasculogenesis. The identification of its downregulation by a simple blood test may stratify patients with high risk to develop EVA. It seems that Klotho has several protective effects with regard to the vasculature and that it represents a promising therapeutic target for preventing oxidative stress of endothelial cells and improving contractility of smooth muscle cells. ${ }^{33}$

Kallistatin, an endogenous protein with protective effects on vessels, has been recently suggested to be used as a powerful inhibitor of cellular senescence and vascular injury. It reduces vascular aging by regulating microRNA34a-SIRT1 pathway. ${ }^{34}$ Moreover, it seems to inhibit telomere shortening and prevent EVA.

\section{Conclusion or future directions? This is the question!}

If we would abruptly make a conclusion of the present review, we should go back to Thomas Sydenham who stated that "A man is as old as his arteries." This is definitely true but, unfortunately, it does not help patients to decrease vascular aging and to improve their life quality. Recently, Cunha et $\mathrm{al}^{36}$ suggested that the dissociation between chronologic and biologic age of large arteries represents the main reason for the failure of proper definition of EVA which overlaps clinical signs and patient prognosis. This paper and others recently published reports ${ }^{35-37}$ focused on a complex 
approach of research focusing on all vascular layers in order to identify specific biomarkers that can be used as predictors of EVA. Histopathology of early steps of vascular aging in prenatal life and in newborns is scattered and incompletely characterized. The microscopic criteria related to age and associated pathologic conditions of the patient are not stated, excepting inconsistent data regarding disorganization of elastic fibers and collagen deposits. There are few studies regarding the molecular mechanisms of EVA with emphasis to the activation of pro-fibrotic, pro-inflammatory, redoxsensitive, and growth/apoptotic signaling pathways, ${ }^{38-40}$ but most of these studies were developed in mice and not yet validated in human subjects.

Complex epigenetic and molecular approach of EVA criteria may lead to an early identification of the process onset and also to apply a personalized therapy dependent on specific biomarkers.

\section{Acknowledgment}

Present work was supported by Romanian Working Group on Atherosclerosis and Atherothrombosis part of Romanian Society of Cardiology.

\section{Disclosure}

The authors report no conflicts of interest in this work.

\section{References}

1. Roguin A. Scipione Riva-Rocci and the men behind the mercury sphygmomanometer. Int J Clin Pract. 2006;60(1):73-79.

2. Cameron JS, Hicks J, Frederick A. Mahomed and his role in the description of hypertension at Guy's Hospital. Kidney Int. 1996;49(5): $1488-1506$

3. Mancia G, Fagard R, Narkiewicz K, et al. ESH/ESC Guidelines for the management of arterial hypertension. Eur Heart J. 2013;34: 2159-2219.

4. Nilsson P, Boutouyrie P, Cunha P. Early vascular ageing in translation a: from laboratory investigations to clinical applications in cardiovascular prevention. J Hypertens. 2013;(31):1517-1526.

5. Nilsson P. Early vascular aging and the role of central BP. J Hypertens. 2011;29:1847-1853.

6. Nilsson P, Boutouyrie P, Laurent S. Vascular aging a tale of EVA and ADAM in cardiovascular risk assessment and prevention. Hypertension. 2009;54:3-10.

7. Doyon A, Kracht D, Bayazit AK. Carotid artery intima-media thickness and distensibility in children and adolescents. Hypertension. 2013; 62:550-556

8. Napoli C, D'Armiento FP, Mancini FP, et al. Fatty streak formation occurs in human fetal aortas and is greatly enhanced by maternal hypercholesterolemia. Intimal accumulation of low density lipoprotein and its oxidation precede monocyte recruitment into early atherosclerotic lesions. J Clin Invest. 1997;100(11):2680-2690.

9. Hodis S, Zamir M. Mechanical events within the arterial wall: the dynamic context for elastin fatigue. J Biomech. 2009;42:1010-1016.

10. Milei J, Ottaviani G, Lavezzi AM, Grana DR, Stella I, Matturri L. Perinatal and infant early atherosclerotic coronary lesions. Can J Cardiol. 2008;24(2):137-141.
11. Bouissou C, Lacolley P, Dabire H. Increased stiffness and cell-matrix interactions of abdominal aorta in two experimental nonhypertensive models: long-term chemically sympathectomized and sinoaortic denervated rats. J Hypertens. 2014;32(3):652-658.

12. Rana S, Pugh PC, Katz E, et al. Independent effects of early-life experience and trait aggression on cardiovascular function. Am J Physiol Regul Integr Comp Physiol. 2016;311(2):R272-R286.

13. Wang Z, Huang Z, Lu G, Lin L, Ferrari M. Hypoxia during pregnancy in rats leads to early morphological changes of atherosclerosis in adult offspring. Am J Physiol Heart Circ Physiol. 2009;296(5): H1321-H1328.

14. Lee SJ, Park SH. Arterial ageing. Korean Circ J. 2013;43(2):73-79.

15. Triantafyllidi $\mathrm{H}$, Trivilou $\mathrm{P}$, Ikonomidis I. Is arterial hypertension control enough to improve aortic stiffness in untreated patients with hypertension? A 3-year follow-up study. Angiology. 2015;66(10): 904-910.

16. Bautista-Niño PK, Portilla-Fernandez E, Vaughan DE, Danser AHJ, Roks AJM. DNA damage: a main determinant of vascular aging. Int $J$ Mol Sci. 2016;17(5):748.

17. Pitale S, Sahasrabuddhe A. Fetal origin of vascular aging. Indian $J$ Endocrinol Metabol. 2011;15(Suppl 4):S289-S297.

18. Coimbra BM, Carvalho CM, Moretti PN, Mello MF, Belangero SI. Stressrelated telomere length in children: a systematic review. $J$ Psychiatr Res. 2017;92:47-54.

19. Steven G, Chrysant SG, Chrysant GS. The age-related hemodynamic changes of blood pressure and their impact on the incidence of cardiovascular disease and stroke: new evidence. J Clin Hypertens. 2014; 16(2):87-90.

20. Ungvari1 Z, Kaley G, de Cabo R, Sonntag WE, Csiszar A. Mechanisms of vascular aging: new perspectives. J Gerontol Biol Sci Med Sci. 2010;65(10):1028-1041.

21. Le J, Zhang D, Menees S, Chen J, Raghuveer G. "Vascular age" is advanced in children with atherosclerosis-promoting risk factors. Circulation. 2010;3:8-14.

22. Pannier B, Guérin AP, Marchais SJ, Michel E, Gérard ML. Stiffness of capacitive and conduit arteries. Hypertension. 2005;45:592-596.

23. Wilkinson I, Cockcroft J, Laurent S, Vlachopoulos C. The reference values for arterial stiffness collaboration - determinants of pulse wave velocity in healthy people and in the presence of cardiovascular risk factors: "establishing normal and reference values." Eur Heart J. 2010; 31:2338-2350.

24. Iurciuc M, Avram C, Iurciuc S, Franculescu C, Vlad A, Mancas S. Comprehensive rehabilitation programs may improve some of the ambulatory blood pressure parameter. Proceedings of the 17th European Congress of Physical and Rehabilitation Medicine. Minerva Medica. 2010:253-255.

25. Iurciuc S, Avram C, Turi V, et al. Physical training, hemodynamic parameters and arterial stiffness: friends or foes of the hypertensive patient? In Vivo. 2016;30(4):521-528.

26. Iurciuc $\mathrm{M}$, Avram $\mathrm{C}$, Iurciuc $\mathrm{S}$, et al. Exercise training and vascular compliance. J Romanian Sports Med. 2007;10:563-568.

27. Laurent S, Cockcroft J, Bortel LV, et al. Expert consensus document on arterial stiffness. Eur Heart J. 2006;27:2588-2605.

28. Mackenzie IS, Wilkinson IB, Cockcroft J. Assessment of arterial stiffness in clinical practice. QJM. 2002;95:67-74.

29. Stabouli S, Papakatsika S, Kotronis G. Arterial stiffness and SBP variability in children and adolescents. J Hypertens. 2015;33(1):88-95.

30. Parati G, Schumacher H, Bilo G, Mancia G. Evaluating 24-h antihypertensive efficacy by the smoothness index: a meta-analysis of an ambulatory blood pressure monitoring database. J Hypertens. 2010; 28(11):2177-2183.

31. Mancia G. Short- and long-term blood pressure variability. Hypertension. 2012;60:512-517.

32. Hajjar I, Hart M, Wan SH, Novak V. Safety and blood pressure trajectory of short-term withdrawal of antihypertensive medications in older adults: experience from a clinical trial sample. J Am Soc Hypertens. 2013;7(4):289-293. 
33. Mencke R, Hillebrands JL; NIGRAM Consortium. The role of the anti-ageing protein Klotho in vascular physiology and pathophysiology. Ageing Res Rev. 2017;35:124-146.

34. Guo Y, Li P, Gao L, et al. Kallistatin reduces vascular senescence and aging by regulating microRNA-34a-SIRT1 pathway. Aging Cell. 2017; 16(4):837-846.

35. Turianikova Z, Javorka K, Baumert M, et al. The effect of orthostatic stress on multiscale entropy of heart rate and blood pressure. Physiol Meas. 2011;32(9):1425-1437.

36. Cunha PG, Boutouyrie P, Nilsson PM, Laurent S. Early vascular ageing (EVA): definitions and clinical applicability. Curr Hypertens Rev. 2017;13(1):8-15.
37. Trindade M, Oigman W, Fritsch Neves M. Potential role of endothelin in early vascular aging. Curr Hypertens Rev. 2017;13(1):33-40.

38. de Moraes R, Tibirica E. Early functional and structural microvascular changes in hypertension related to aging. Curr Hypertens Rev. 2017; 13(1):24-32.

39. Harvey A, Montezano AC, Touyz RM. Vascular biology of ageing implications in hypertension. J Mol Cell Cardiol. 2015;83:112-121.

40. Harvey A, Montezano AC, Lopes RA, Rios F, Touyz RM. Vascular fibrosis in aging and hypertension: molecular mechanisms and clinical implications. Can J Cardiol. 2016;32(5):659-668.
Clinical Interventions in Aging

\section{Publish your work in this journal}

Clinical Interventions in Aging is an international, peer-reviewed journal focusing on evidence-based reports on the value or lack thereof of treatments intended to prevent or delay the onset of maladaptive correlates of aging in human beings. This journal is indexed on PubMed Central, MedLine,

\section{Dovepress}

CAS, Scopus and the Elsevier Bibliographic databases. The manuscript management system is completely online and includes a very quick and fair peer-review system, which is all easy to use. Visit http://www.dovepress. com/testimonials.php to read real quotes from published authors. 